\title{
Scale-Free Correlations in Flocking Systems with Position-Based Interactions
}

\author{
Cristián Huepe • Eliseo Ferrante • Tom Wenseleers • \\ Ali Emre Turgut
}

Received: 16 April 2014 / Accepted: 9 September 2014

C) Springer Science+Business Media New York 2014

\begin{abstract}
We consider a model of self-propelled agents with spring-like interactions that depend only on relative positions, and not on relative orientations. We observe that groups of these agents self-organize to achieve collective motion (CM) through a mechanism based on the cascading of self-propulsion energy towards lower elastic modes. By computing the correlation functions of the speed and velocity fluctuations for different group sizes, we show that the corresponding correlation lengths are proportional to the linear size of the group and have no intrinsic length scale. We argue that such scale-free correlations are a natural consequence of the position-based interactions and associated CM dynamics. We hypothesize that this effect, acting in the context of more complex realistic interactions, could be at the origin of the scale-free correlations measured experimentally in flocks of starlings, instead of the previously argued proximity to a critical regime.
\end{abstract}

Keywords Collective motion · Flocks - Swarms - Scale-free correlations - Criticality · Self-organization · Active matter · Active elastic systems · Energy cascade

\section{Introduction}

Collective motion (CM) is observed in a broad range of biological systems, including bird flocks, fish schools, herds of quadrupeds, insect swarms, and groups of bacteria $[8,14,21$, $25,33,42]$. In recent years, systems of self-propelled agents displaying CM (referred to here, generically, as flocking systems) have been the subject of intense research [16,35,41]. Several

\author{
C. Huepe ( $\varangle)$ \\ CHuepe Labs, 954 W. 18th Place, Chicago, IL 60608, USA \\ e-mail: cristian@northwestern.edu \\ C. Huepe \\ Northwestern Institute on Complex Systems, Northwestern University, Evanston, IL 60208, USA \\ E. Ferrante · T. Wenseleers · A. E. Turgut \\ Laboratory of Socioecology and Social Evolution, KULeuven, Naamsestraat 59, 3000 Leuven, Belgium
}


models have been introduced to analyze their dynamical properties, their ability to process information collectively, and the components required to achieve CM. Recent research in control theory and robotics has also focused on CM, developing various decentralized control algorithms that mimic flocking dynamics to achieve a similar level of coordinated collective behavior in groups of autonomous robots [7,19,39,41].

From the perspective of nonequilibrium statistical physics, flocking systems uniquely combine familiar spatial dynamics with novel phenomena. On the one hand, they can be modeled as an ensemble of particles that have an internal source of kinetic energy and interact through effective forces. On the other hand, these interactions could be non-central, non-additive, or non-local; they could be based on positions, velocities, accelerations, or even trajectories; and they could be processed to affect individual motion in any number of ways. These features and a growing number of experiments and potential applications have made flocking systems an exciting new field of research at the interface between physics, biology, and engineering. Despite intense recent research activity, however, there is still no comprehensive understanding of all underlying mechanisms that can lead a group of selfpropelled agents to self-organize and move in a common direction.

The prevailing paradigm in the theory of CM has been strongly influenced by the seminal work of Vicsek et al. [40], which introduced a minimal model for flocking, the Vicsek model, that has become a point of reference in the field [7,16,41]. This model describes a group of point particles advancing at a fixed common speed, only coupled through explicitly aligning interactions that steer each particle towards the mean heading direction of all neighbors within a given radius $[15,40]$. The system undergoes a dynamical phase transition at a critical noise level, below which particles self-organize to move in a common direction, thus displaying CM. In this framework, a flock is viewed as a fluid of self-propelled spins with aligning interactions, described by an extension of the XY-model [6] where spins advance in their pointing direction rather than remaining affixed to a lattice. Individuals are modeled as pointlike objects without attractive or repulsive interactions, so relative positions have no influence on the dynamics except for determining which particles are close enough to interact. Taking a perspective similar to the Vicsek model, but using a continuous description rather than agentbased dynamics, Toner and Tu introduced a hydrodynamic theory of active fluids [37,38]. In this approach, a generalized form of the Navier-Stokes equation is formulated for a fluid with self-driven advection, which includes additional terms due to the lack of Galilean invariance. This framework describes a very general situation and can include any equation of state, but it does not consider elastic-like interactions. Therefore, both this continuous approach and the Vicsek model could miss any effect related to the position-based interactions that must be associated to any level of rigidity in flocking structures or formations.

Various alternative models of self-propelled agents that produce CM have been introduced in the literature, some of which are position-based and require no explicitly aligning interaction [41]. In [34], for example, CM is driven only by escape-pursuit dynamics; in [24], by the conservation of momentum in inelastic collisions between isotropic self-propelled agents; in [31], by the deformation of self-propelled soft spherical particles with local repulsion; and in $[26,36]$, by short-range radial repulsion forces coupled to the agents' turning dynamics. While it can be argued that some of these algorithms result in effective interactions that are equivalent to explicit alignment and thus belong to the same universality class as the Vicsek model, others appear to produce fundamentally different dynamics, which can display, for example, configurations where pairs of agents are driven away from alignment. We will focus below on one of these alternative models; a minimal model recently introduced in $[17,18]$ to describe an idealized active elastic (AE) system that corresponds in many ways to a limit case opposite to the Vicsek model. While in the Vicsek algorithm interactions depend exclusively 
on the relative orientation of neighbors, in the AE case they depend only on their relative positions. While in the continuous limit the Vicsek model can be viewed as an active hydrodynamic system, the AE model can be viewed as an unbreakable elastic membrane with self-propelled components (since agents are permanently linked to their neighbors by virtual springs). As in the Vicsek model, the AE model also converges to CM, but it does so through an elasticity-based mechanism that is fundamentally different from the ferromagnetic-like aligning dynamics that leads to CM in the Vicsek case. We note that the AE model is similar to the model in [36] and [26]. We chose to focus here on the AE model, however, because: (1) attraction-repulsion forces between agents in $[26,36]$ can displace them perpendicular to their heading directions, which does not properly describe the flocking dynamics, and (2) the mechanism that leads the AE algorithm to CM was identified and well-studied in $[17,18]$.

The question of what are the actual interactions between individuals in different animal groups that display flocking behavior is still an open one. The answer probably depends on the species and on the specific dynamical regime considered. These interactions could be based on information that cannot be directly expressed in terms of the usual, position and velocity, physical variables. Agents could be using memory and/or prediction, which involves additional knowledge of their trajectories. They could also be receiving specific sensory inputs that are a complex function of the spatial configuration. Vision, for example, includes the effects of screening, visual field, and feature visibility. It is reasonable to expect, however, that in many cases the position-based and orientation-based interactions described above can serve as a proxy for the actual, more complex, underlying interactions between biological individuals, and that they can thus effectively capture at least some of the essential features of CM.

In recent years, various experimental efforts have tried to unveil the interactions used by different organisms to achieve CM [3,9-11,22,23,27,30]. In experiments with small groups of fish (golden shiners), it was shown that individuals react mainly to each other's relative positions, and more weakly to other less standard quantities such as the group speed [27]. No evidence was found, however, of interactions that would tend to align the relative heading angles of neighbors. In contrast, general arguments lead us to expect that positionbased interactions should always be relevant for CM dynamics. Indeed, repulsion forces are required to avoid collisions and attraction forces must play a role in achieving group formation in the first place. All these factors suggest that alignment-based interactions may not be the main mechanism behind the flocking dynamics of many animal groups.

Despite the lack of experimental evidence showing that aligning interactions are dominant in the CM of real animal groups, several studies have assumed that these are the only relevant interactions when modeling their dynamics $[3,5,9,11]$. This has been the case, in particular, in series of papers that analyzed the three-dimensional trajectories of individuals in starling flocks obtained by implementing sophisticated tracking and data-analysis techniques [3, $11,12]$. These trajectories were studied using various theoretical approaches to deduce the underlying interactions between birds, using the data to deduce the corresponding social forces and effective Hamiltonians, but always assuming that the interaction terms depend almost exclusively on the relative orientation between individuals. This somewhat restrictive approach appears to be driven by the enticing analogies that can be established between alignment-driven flocks and the XY-model, a system that has been well-studied in statistical physics.

One of the most surprising results that was obtained from the analysis of the starling data is that the correlation lengths of the birds' speed and velocity fluctuations are not set by specific properties of the interactions, but are instead scale-free and proportional to the 
linear group size (defined as the maximum distance between two birds in the flock) [12]. This observation prompted the speculation that flocks may adjust their dynamics to pose themselves close to a phase transition, since such scale-free correlations are expected in finite systems near a critical regime. For example, the Abstract of [12] states that the results presented in that paper "suggest that flocks behave as critical systems, poised to respond maximally to environmental perturbations". If proven true, this could relate flocking dynamics to the concept of life at the edge of chaos [2,28,29,32], which postulates that evolution drives living systems towards a critical state where they can either follow robust dynamics, to resist perturbations and localized failure, or access chaotic dynamics that can rapidly explore new possibilities, if needed. We will argue below, however, that there may be an alternative, more parsimonious explanation for the observed scale-free correlations that does not involve criticality, by presenting an example of a $\mathrm{CM}$ algorithm with position-dependent interactions (the AE model) that naturally displays such correlations far from any critical regime. Note that we focus here on algorithms that could describe bird flocks and that do not contain intrinsically long-range interactions, since these could trivially lead to scale-free correlations, as shown in [4], for example, for groups of swimmers with long-range hydrodynamic interactions.

In this paper, we show that the AE model produces scale-free correlation lengths that are proportional to the group size, even when the system is far from any critical regime. Although this model is highly idealized, we argue that the mechanism that produces scalefree correlations in our simulations could lead to the same effect in experimental systems. The paper is organized as follows. In Sect. 2, we introduce the AE model. Section 3 describes the energy cascading mechanism that drives the AE dynamics towards $\mathrm{CM}$, and shows that its convergence process will tend to display scale-free correlations. In Sect. 4, we present simulations that mimic the starling experiment, in which we obtain the same type of scalefree correlations despite being far from any critical state. Finally, Sect. 5 discusses our results and presents our conclusions.

\section{The Active Elastic Model}

We consider a system of $N$ self-propelled agents moving on a two-dimensional plane. Neighboring agents are linked by spring-like forces affecting their speeds and heading angles. The AE model is defined by the following set of overdamped dynamical equations for the position vector $\mathbf{x}_{i}$ and orientation $\theta_{i}$ of each agent $i$

$$
\begin{aligned}
\dot{\mathbf{x}}_{i} & =v_{0} \hat{n}_{i}+\alpha\left(\mathbf{F}_{i} \cdot \hat{n}_{i}\right) \hat{n}_{i}, \\
\dot{\theta}_{i} & =\beta\left(\mathbf{F}_{i} \cdot \hat{n}_{i}^{\perp}\right)+D_{\theta} \xi_{\theta} .
\end{aligned}
$$

Here, $v_{0}$ is the preferred self-propulsion speed (equal for all agents), $\alpha$ and $\beta$ are the inverse translational and rotational damping coefficients, respectively, $\hat{n}_{i}$ is a unit vector that points in the heading direction of agent $i$ while $\hat{n}_{i}^{\perp}$ is a unit vector that points perpendicular to it, and $\mathbf{F}_{i}$ represents the sum of elastic forces over agent $i$. Noise is introduced by adding $D_{\theta} \xi_{\theta}$ to the heading angle equation, where $D_{\theta}$ is the noise strength and $\xi_{\theta}$ a random variable with standard, zero-centered normal distribution of variance 1 . These equations have a simple interpretation: each agent adds to its self-propulsion speed the projection of elastic forces along its heading direction and turns according to the projection of elastic forces perpendicular to its heading direction. 
The total elastic force $\mathbf{F}_{i}$ over agent $i$ is given by the sum of linear spring-like forces

$$
\mathbf{F}_{i}=\sum_{j \in S_{i}}-\frac{k}{l_{0}}\left(\left\|\mathbf{r}_{i j}\right\|-l_{0}\right) \frac{\mathbf{r}_{i j}}{\left\|\mathbf{r}_{i j}\right\|},
$$

where $\mathbf{r}_{i j}=\mathbf{x}_{j}-\mathbf{x}_{i}$. Each set $S_{i}$ contains the $j$ indexes of all agents linked to agent $i$ by virtual springs with spring constant $k / l_{0}$ and natural length $l_{0}$. These links are unbreakable, so the $S_{i}$ sets remain unchanged throughout the simulation. We note that the AE model is similar to a spring-mass model of an elastic sheet [20], where inert masses are replaced by self-propelled agents restricted to only turn or move along their heading direction.

We carried out simulations by implementing a standard Euler method, integrating Eqs. (1) and (2) numerically as detailed in [17]. We will consider below two different simulation setups. The first one (Sect. 3) illustrates the elasticity-driven mechanism that drives the AE dynamics to CM. We will show that this mechanism, in itself, favors collective oscillations that can result in scale-free correlations. The second setup (Sect. 4) uses the AE model to implement a rough cartoon of the experimental dynamics of starling flocks reported in [12]. We will show that correlation lengths grow with the linear group size, as in the experiments, despite considering simulations that are far from any critical regime.

Although our intention was not to model the experiments in detail, we chose a set of parameters that roughly mimics their dynamics in order to facilitate comparisons to the experimental results. We thus set the equilibrium distance between neighbors to $l_{0}=1$ and the self-propulsion speed to $v_{0}=10$ in all simulations below, since the mean distance between neighboring starlings and the mean starling speed were reported to be of the order of $1 \mathrm{~m}$ and $10 \mathrm{~m} / \mathrm{s}$, respectively [12]. Noise was set to zero for the simulations in Sect. 3, to focus on illustrating the self-organizing dynamics. The noise level in Sect. 4 was set to be high, yet far from the transition point, to mimic a generic experimental situation. The remaining parameters were chosen to produce variances in the speed and heading angles that are compatible with the experimental values. Note that the connection between our simulations and the experiments is too loose to warrant a detailed matching of simulation parameters. Instead, we argue that the displayed $\mathrm{AE}$ dynamics is generic enough to qualitatively match some of the experimental features without requiring any fine-tuning of the parameters.

\section{Energy Cascading Mechanism}

We will show in this section that the same energy cascading mechanism that leads the AE model to self-organize into $\mathrm{CM}$ will also favor the excitation of collective oscillatory modes, which results in turn in scale-free correlations.

We begin by computing the elastic energy of an AE model simulation and how it is distributed among the different elastic modes. In order to do this, we first need to find the elastic normal modes of the system, which was done numerically by evaluating the elasticity matrix of the simulated AE structure (by measuring the effect of perturbing each agent's position with respect to equilibrium) and computing its eigenvectors. Note that these normal modes only consider elastic deformations; they do not include the self-propulsion dynamics or the motion constraints imposed by the model (where agents can only move forward, backwards, or turn). However, they can be used as an orthogonal basis where we expand the deformation of the hexagonal structure to find the energy content of each mode. We point out that the total energy is not conserved here, since it is being injected through the 

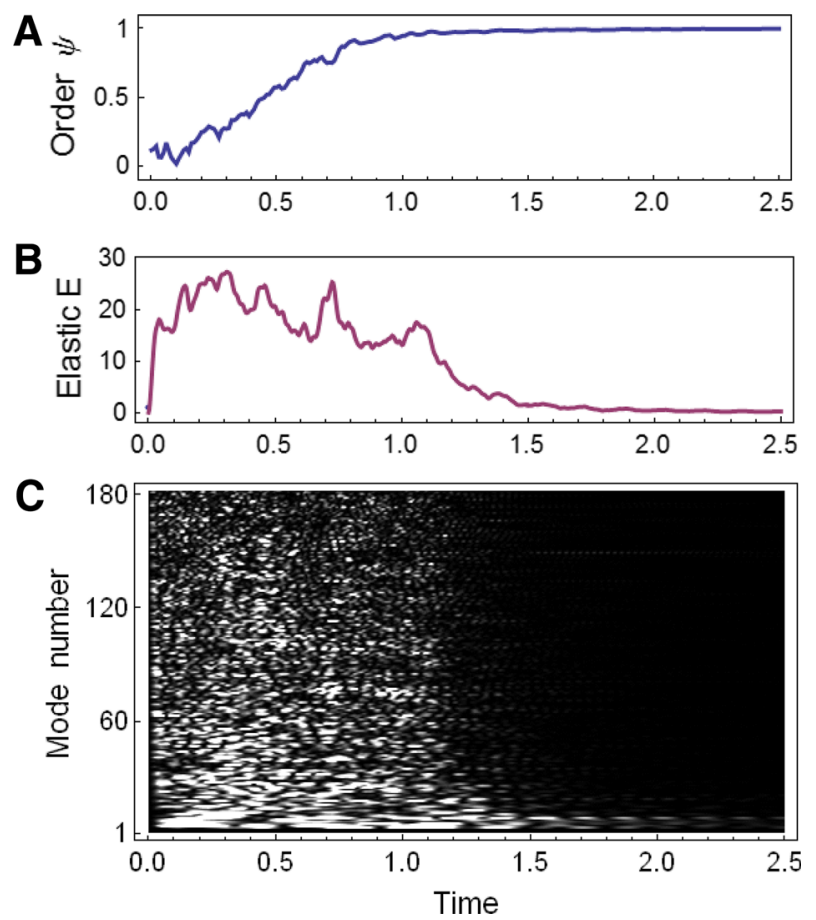

Fig. 1 Order parameter $\psi$ (a) defined in Eq.(9), elastic energy (b), and spectral decomposition of the elastic energy (c) as a function of time for an $\mathrm{AE}$ simulation with $N=91$ agents and zero noise. The spectral decomposition is displayed over all 182 elastic normal modes, presented in order of growing energy, with brighter points indicating a larger mode energies. After a transient, the total elastic energy converges to zero and agents align, as indicated by the order parameter. Despite the oscillatory nature of the modes' decays (which results in the displayed speckled distribution of energy over time) and complex interplay between them, it is apparent that higher elastic modes dampen out before lower ones. During the second half of the run $(t>1.2)$, most of the energy has been transferred to lower modes, generating (in addition to CM) collective oscillations that result in scale-free correlations

self-propulsion speed $v_{0}$ while it is continuously dissipated due to the overdamped nature of the equations.

Figure 1 shows the elastic energy dynamics as a function of time for an AE simulation with 91 self-propelled agents forming an hexagonal structure. Agents were initially placed with random headings on a regular hexagonal lattice configuration. The distance between nearest neighbors and the natural length of the virtual springs that link them were both set to $l_{0}$, so the elastic structure was initially undeformed. Model parameters were set to: $l_{0}=1, v_{0}=10$, $\alpha=2, \beta=3$, and $D_{\theta}=0$. The numerical integration timestep was set to $\Delta t=10^{-4}$. Panel A displays the total elastic energy as a function of time. Panel B shows how this energy is distributed among the 182 existing modes (one per agent and per degree of freedom), which are presented here in order of growing energy. Initially, the elastic energy is zero, since all springs start at their equilibrium length. As the system evolves, agents start moving in their random initial headings, exciting all elastic modes and increasing the total elastic energy. At $t \approx 1.2$, most higher elastic modes have decayed and the self-propulsion energy has focused on either CM or collective oscillations. After $t \approx 1.5$, the elastic energy has almost fallen to zero as all agents have become aligned and regained their equilibrium distances, now moving in parallel towards a common direction. 
The mechanism behind the self-organizing dynamics described above can be understood through an analogy with a standard damped vibrating system. In such systems, higher elastic modes dissipate faster, since it takes more energy to excite them, thus making the lower elastic modes the last ones to damp out. This familiar phenomenon is the reason why we end up hearing the fundamental note when picking a string or striking a drum, despite initially exciting a broad superposition of modes. A similar phenomenon is occurring here, but in this active system case it leads to CM. Instead of dissipating faster, agents steer away from higher elastic modes due to the coupling between headings and elastic forces. This produces an energy cascading mechanism that transfers the self-propulsion energy to lower and lower elastic modes, eventually reaching the translational or rotational modes and achieving CM $[17,18]$

It is apparent on Fig. 1 that residual fluctuations after the system achieves CM typically consist of collective oscillation modes (with low mode number on panel B) that involve the whole group. Indeed, we observe that after $t \approx 1.2$ higher elastic modes have mostly dampened out and only lower ones remain. These lower modes decay slowly while displaying underdamped oscillations. Collective oscillation modes, which produce correlation lengths that scale with the size of the system, are therefore a natural consequence of the AE model's convergence dynamics towards CM. However, this simulation was not set up to explore the type of flocking dynamics studied in [12], since it starts with random orientations and includes no noise or perturbations. We will show below that collective oscillations, and therefore scale-free correlations, are in fact ubiquitous in the AE model when we include some generic features of flocking dynamics.

\section{Perturbations and Scale-Free Correlations}

In this section, we will set up simulations of the AE model that roughly mimic the flocking dynamics analyzed experimentally in [12]. In that study, snapshots of the positions and velocities of all individuals in flocks of between 122 and 4,268 starlings (freely flying in three dimensions) were used to show that the correlation lengths of their speed and velocity fluctuations are proportional to the linear size of the group (the largest distance between two birds in the flock). We carried out AE simulations of groups of between 91 and 4,681 agents to generate a similar dataset and analyze the corresponding correlation lengths.

In order to address the type of fluctuation dynamics that is observed in real, freely flying flocks, we must consider the fact that these are continuously changing direction due to strong perturbations of the group heading induced by a few individuals. Recent experimental analyses have shown that these turns are typically initiated by a single bird at the edge of the flock [1], most likely in response to external stimuli. We set up simulations that imitate this kind of dynamics by considering groups that are moving collectively, but where one of the agents has selected a new heading direction.

The simulations are prepared as follows. For each group size, we first set up a twodimensional hexagonal lattice as in Sect. 3, initially placing all agents on the undeformed structure, pointing towards $\theta=0$ (along the x-axis), and run the dynamics until a steady state of the order parameter is reached. Then a single agent, which we will refer to as the informed agent, is turned at a time we define as $t=0$ to point to a new $\theta=\pi / 3$ heading direction, which is imposed as its heading direction for the rest of the simulation while its speed is left to evolve according to Eq. (1), as usual. The informed agent is selected at the edge of the group, in a way consistent with the experimental results in [1]. 
We analyzed the resulting dynamics following the same approach used in [12]. First, we computed the residual velocities and speeds by subtracting their mean values. The residual velocities are thus given by

$$
\mathbf{u}_{i}=\mathbf{v}_{i}-\frac{1}{N} \sum_{i=1}^{N} \mathbf{v}_{k}
$$

and the residual speeds, by

$$
s_{i}=\left\|\mathbf{v}_{i}\right\|-\frac{1}{N} \sum_{i=1}^{N}\left\|\mathbf{v}_{k}\right\|,
$$

where $\mathbf{v}_{i}$ is the velocity of agent $i$. The correlation functions for $\mathbf{u}_{i}$ and $s_{i}$ were then evaluated at selected timeframes, using the expressions

$$
C_{\mathbf{u}}(r)=A \frac{\sum_{i, j=1}^{N} \mathbf{u}_{i} \cdot \mathbf{u}_{j} \delta\left(r-r_{i j}\right)}{\sum_{i, j=1}^{N} \delta\left(r-r_{i j}\right)}
$$

and

$$
C_{s}(r)=B \frac{\sum_{i, j=1}^{N} s_{i} s_{j} \delta\left(r-r_{i j}\right)}{\sum_{i, j=1}^{N} \delta\left(r-r_{i j}\right)} .
$$

Here, $A$ and $B$ are normalization constants defined so that $C_{\mathbf{u}}(0)=C_{s}(0)=1, r_{i j}$ is the distance between agent $i$ and agent $j$, and $\delta(\cdot)$ is a smoothed Dirac delta function, which we define by

$$
\delta(r)=\frac{1}{\sqrt{2 \pi} \sigma} \exp \left(\frac{-r^{2}}{2 \sigma^{2}}\right) .
$$

While the exact choice of $\sigma$ does not change the qualitative features of the resulting curves, we used $\sigma=l_{0} / 2$ since it is the smallest value that produces smooth correlation functions.

Figure 2 displays some of the characteristic features of a typical simulation snapshot. Here, an hexagonal structure made up of $N=1,519$ self-propelled agents was initialized as described above, with the top-right vertex chosen as the informed agent in order to be consistent with the experimental observations in [1]. Note that the informed agent's imposed $\theta=\pi / 3$ corresponds here to a heading angle that is pointing radially outwards with respect to the center of the group at $t=0$. Here, $\theta=0$ is along the $\mathrm{x}$-axis (towards the right on panel A) and angles grow counterclockwise. Simulations were carried out using the same model parameters as in Sect. 3, with the exception of the noise level, which was set to $D_{\theta}=0.628$. This value was chosen to be relatively high, yet far from the critical point $D_{\theta}^{*} \approx 1.1$, where the system transitions to a disordered, non-flocking state. The data presented corresponds to a simulation snapshot taken at $t=1.9$, a somewhat arbitrary moment in time that we chose for evaluating the correlation functions of all simulations presented in this section. It was selected in order to have a common instant to compare all setups that was slightly after the time required for the informed agent's perturbation to cross our largest $(N=4681)$ hexagonal structure. Since we are mimicking a single perturbation event, if we considered much earlier times most of the group would still be unaffected by the informed agent and at much later times the perturbation will have decayed. Equivalent results were obtained, however, for snapshots taken within a broad range of times.

Panel A displays a snapshot of the spatial dynamics, with the velocity $\mathbf{v}_{i}$ of each agent represented by a small vector. It shows the group turning slightly upwards, as indicated by the large overlaid arrow, which points in its mean heading direction. The informed agent appears as detached from the hexagon, above and to the left of its top-right vertex, since its 

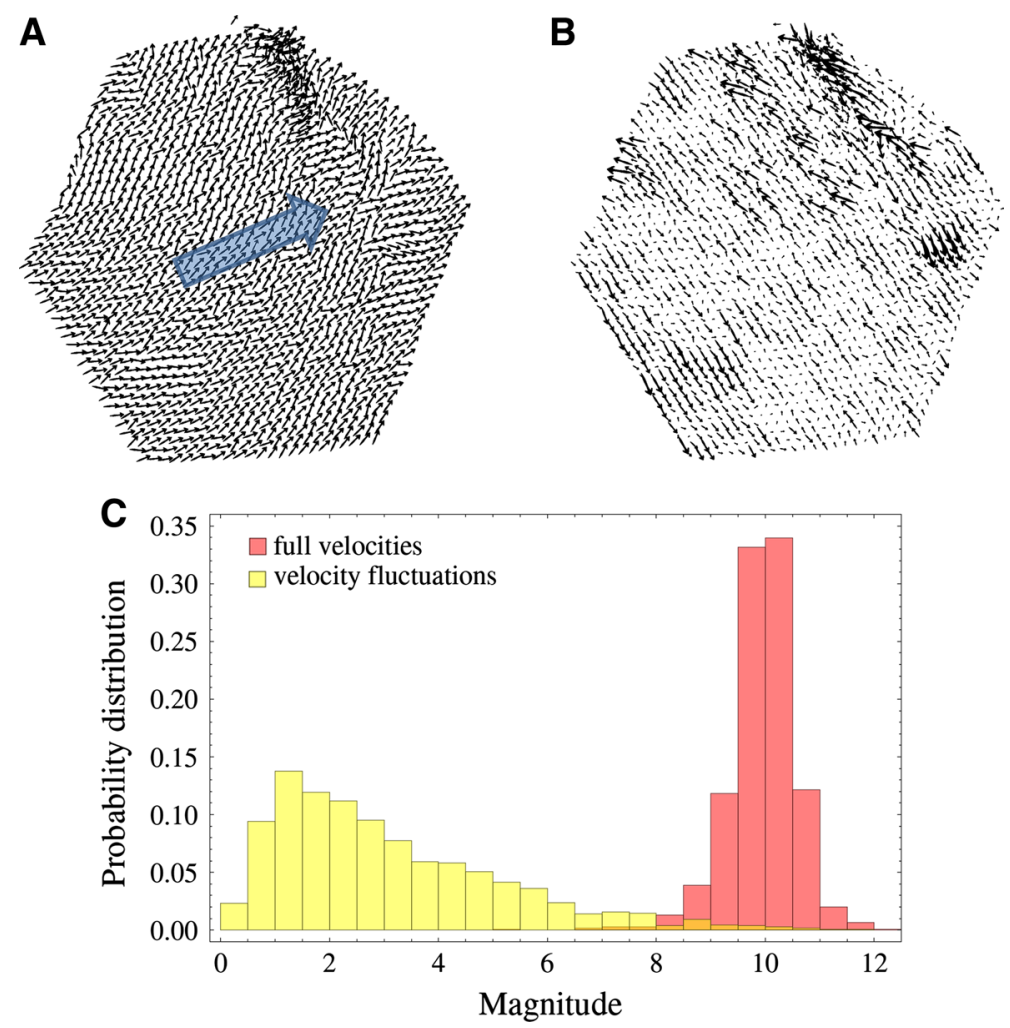

Fig. 2 Simulation data at $t=1.9$ of an hexagonal AE structure containing $N=1,519$ self-propelled agents. a Snapshot showing the velocity $\mathbf{v}_{i}$ of each agent, with a large overlaid arrow indicating the group's mean heading direction. b Velocity fluctuations $\mathbf{u}_{i}$, where large domains of correlated motion are visible. $\mathbf{c}$ Normalized distributions of the magnitude of individual velocities and velocity fluctuations

virtual springs are significantly stretched due to the large forces that they must sustain as they make the whole group turn. Agents display clear deviations from perfect alignment because of the local perturbation and imposed noise. The degree of alignment is given by the standard polarization order parameter

$$
\psi=\frac{1}{N v_{0}}\left\|\sum_{i=1}^{N} \mathbf{v}_{i}\right\|,
$$

which is equal to 1 if all agents are perfectly aligned and to 0 if they have no preferred heading direction. The displayed snapshot has $\psi \approx 0.93$, a high level of alignment that is consistent with the $0.886 \leq \psi \leq 0.995$ range of polarization values reported in the starling experiments [12]. Panel B shows the residual velocities $\mathbf{u}_{i}$, computed using Eq. (4). Large fluctuations are observed in the region close to the informed agent. Despite this strong localized perturbation, the group also displays large regions of coherent motion over which the residual velocity vectors are similar to each other. Panel $\mathrm{C}$ presents the distributions of the magnitude of the full and residual velocities displayed on panels A and B. As expected, residual velocities are typically much smaller, since the center of mass velocity was subtracted from them. All three panels of Fig. 2 can be compared to the corresponding panels in the first figure of reference [12], which was obtained directly from experimental measures of groups of starlings. We 

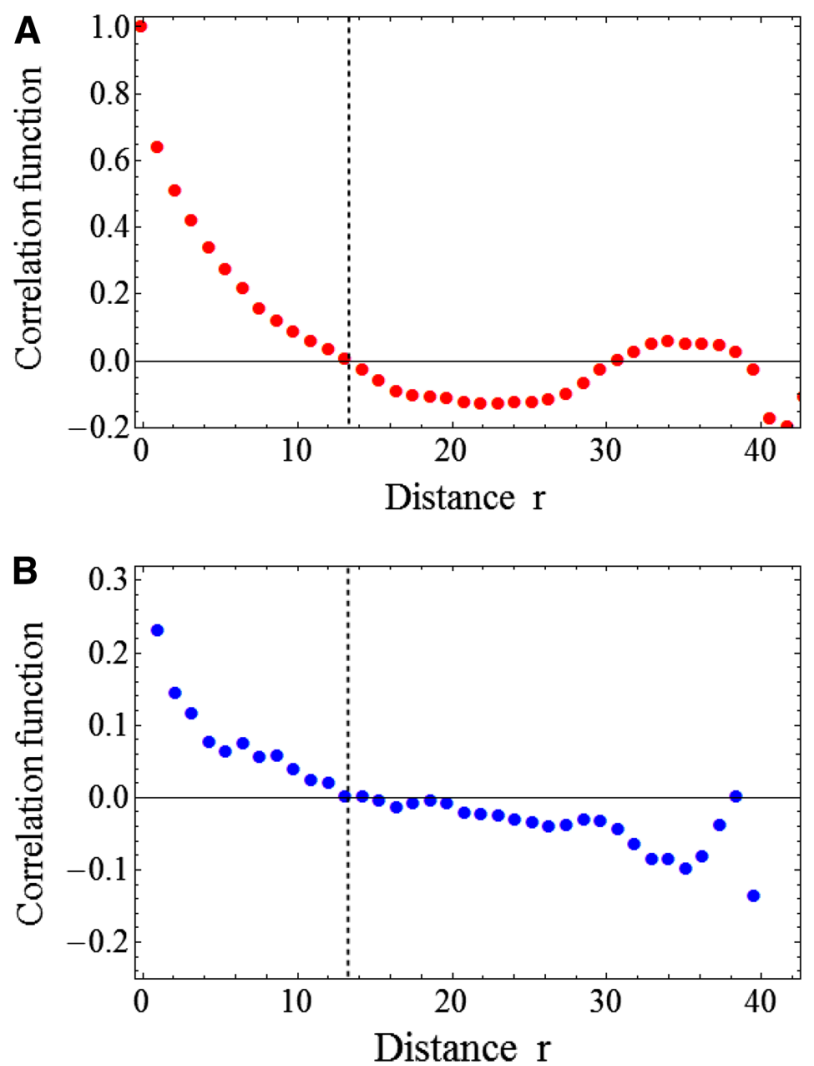

Fig. 3 Correlation functions of the velocity fluctuations $\mathbf{u}_{i}$ (a) and speed fluctuations $s_{i}$ (b) for the same snapshot presented in Fig. 2. The first zero-crossing point in each case (indicated by a dashed vertical line) defines the corresponding correlation length. Both correlation lengths are much larger than the interaction range $l_{0}=1$ and a significant fraction of the linear size of the group $L=45$

observe that both the experiments and our AES simulations display qualitatively equivalent dynamics.

Figure 3 displays the velocity and speed correlation curves, $C_{\mathbf{u}}(r)$ and $C_{s}(r)$, of the same snapshot presented on Fig. 2. We find that they are similar to the experimental correlation curves obtained for starling flocks (see Fig. 2 in Ref. [12]). In particular, their corresponding correlation lengths are also a significant fraction of the size of the whole group. We define these correlation lengths as the first zero-crossing point of $C_{\mathbf{u}}(r)$ and $C_{S}(r)$ (indicated on the plots by a dashed vertical line). Note that these curves must cross zero, since $\mathbf{u}_{i}$ and $s_{i}$ are defined to have zero mean value.

We observe on Fig. 3 that a difference between the displayed simulation-based correlation curves and the experimental ones is that the former appear to decay somewhat faster close to $r=0$ than the latter. This could be due to the lack of explicit aligning interactions in the AE model, which would enhance these correlations and produce a slower decay. It could also be due to the fact that our simulations are two-dimensional while the experiments occur in three dimensions, where a group with the same number of agents has a smaller linear size. It could result from the idealized single informed agent dynamics that we considered. Furthermore, we observed that this initial decay is typically less pronounced in our smaller 

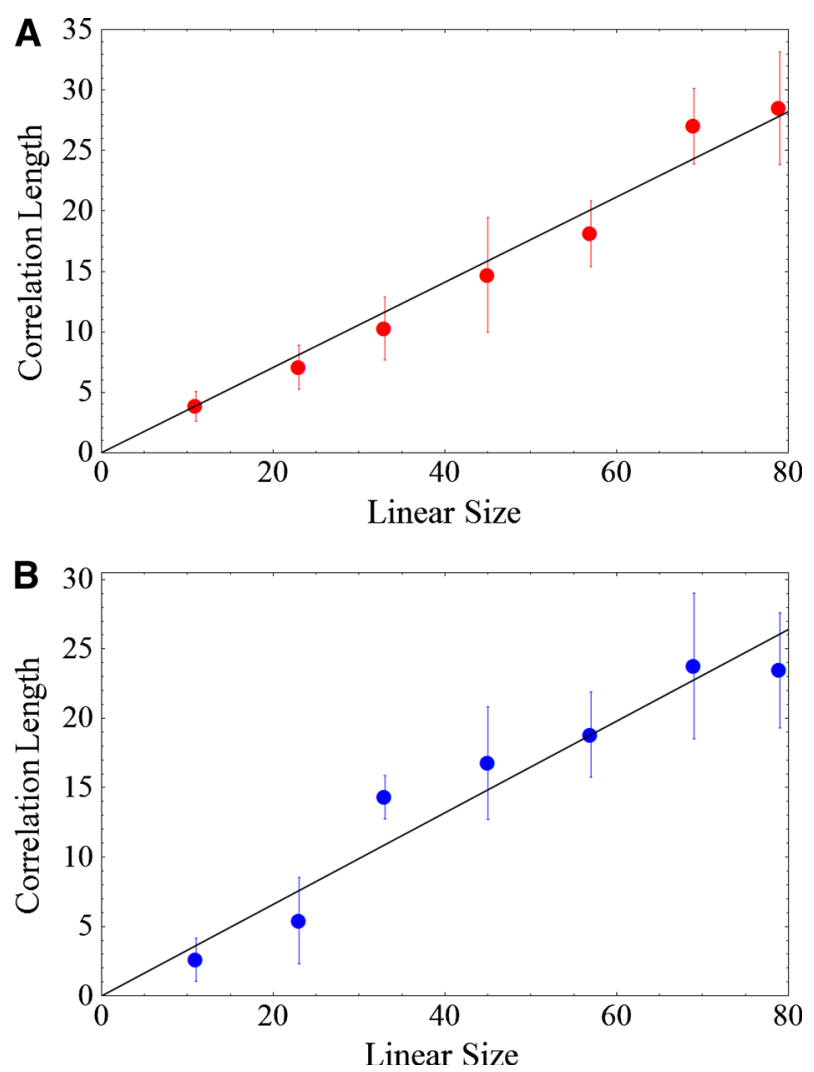

Fig. 4 Correlation lengths of the velocity fluctuations $\mathbf{u}_{i}(\mathbf{a})$ and speed fluctuations $s_{i}(\mathbf{b})$ as a function of the linear group size $L$ for simulations of hexagonal AE structures containing between $N=91$ and $N=4,681$ self-propelled agents (see text). All other parameters are the same as in Figs. 2 and 3. Each point displays the mean correlation length, averaged over six snapshots taken between $t=1.9$ and $t=2.4$. Error bars show the corresponding standard deviations. The lines represent linear fits. Correlation lengths are found to be proportional to the size of the group and, therefore, scale-free

systems (data not shown) and that the exact shape of these curves fluctuates strongly with time. This difference could thus depend on the size and shape of the group and on the specific snapshot chosen. Either way, such difference is not surprising given the idealized nature of our simulations. A more important point in the context of our current analysis is the qualitative agreement between the correlation lengths measured in these minimal simulations and in the experiments.

In order to analyze how the correlation lengths scale with system size, we carried out the same type of simulations described above for groups of $N=91,397,817,1519,2437$, 3571 , and 4681 agents. These numbers were chosen because they produce perfect hexagonal structures and they cover a range of group sizes similar to the starling data. Figure 4 presents the corresponding correlation lengths as a function of linear group size, defined here as the maximum distance between two agents in the undeformed hexagonal structure. Each point shows the mean correlation length averaged over six snapshots, taken at $t=1.9,2.0,2.1,2.2$, 2.3, and 2.4. Error bars display their standard deviation. Although the exact values fluctuate with time, the typical relationship between the correlation length and system size is clearly 
linear. This is the same relationship that was observed in the experimental data reported in [12] (see, in particular, panels C and D of Fig. 2 in that paper). We have thus shown that the initially surprising experimental feature of having scale-free correlations in the speed and velocity fluctuations of starling flocks can be reproduced using a minimal model with position-based interactions, where it requires no critical dynamics and no specific fine-tuning of the parameters or of the interaction topology.

\section{Discussion and Conclusions}

The results above show that the scale-free correlations of speed and velocity fluctuations measured in starling flocks [12] could result from collective deformation modes of the structure of the group when position-dependent interactions are considered. This stems from the simple fact that an elastic solid will typically tend to rotate or deform following its lower elastic modes, since this requires less energy than exciting higher modes. To favor such collective excitations in an active system, however, the self-propulsion energy involved in local deformations must be able to cascade to lower modes. In our AE model, such cascading mechanism is precisely what is behind its ability to self-organize into CM despite lacking explicit alignment rules, as shown in Sect. 3. Local perturbations thus quickly cascade towards collective deformation modes in our AE simulations, where they can produce persistent excitations that then result in the observed scale-free correlations.

The AE model allowed us to study independently the effect of simple position-based interactions, which had been completely neglected in most previous analyses. Our purpose, however, was not to specifically model the starling experiments, since we considered an idealized situation that does not adequately describe starling flocks. Firstly, the AE model includes no interactions that depend on relative orientations, whereas real birds must be aware of their neighbors' heading directions. Secondly, our simulations are based on semirigid agent formations in which interacting neighbors do not change throughout the dynamics. By contrast, starling flocks quickly lose their structure and display strong mixing as they fly together, with birds constantly changing their local neighborhood. Finally, our simulations were carried out in two dimensions while the experimental dynamics is three-dimensional. Despite these idealizations, we argue that the mechanisms observed in our minimal AE model could also play an essential role in generating the experimentally observed scale-free correlations.

The fact that a minimal AE model with generic parameters displays scale-free correlations that are qualitatively equivalent to the experimental results suggests that position-based, attraction/repulsion-like interactions can lead to a robust mechanism for generating such dynamics. Future studies should determine if this mechanism will remain present in more realistic setups. We plan to examine the effects of including aligning interactions based on relative angles, of considering soft attraction-repulsion potentials that allow fluid-like mixing, and of implementing three-dimensional simulations. Preliminary results suggest, however, that the presence of these additional factors will not destroy the core scale-free correlation effect described in this work.

Our findings provide an alternative mechanism for producing the scale-free correlations reported in [12] without having to appeal to the presence of critical dynamics. While criticality may be a fascinating feature of living systems, supported by powerful fundamental arguments on the robustness and adaptability required for evolution, when searching for it in experiments it is important to carefully consider other mechanisms that could produce similar signatures in complex finite systems. In the current case, our results suggest that the scale-free dynamics 
observed in starling flocks may not require a subtle explanation like the development of critical dynamics, but could instead be simply due to the presence of position-based interactions that had been completely neglected in some of the previous models. We note that more recent publications based on the same data provide further arguments for the critical dynamics of starling flocks $[4,5,13]$, but that they all neglect position-based interactions and could thus also overlook the effects discussed here. In particular, it is shown in [4] (published after the initial submission of this paper) that a Hamiltonian with only velocity-based interactions fits the data correctly when its control parameter is poised near a critical point and the boundary conditions of the flock are matched to the experiments. In our view, however, this result could be a consequence of imposing a model that can only display long-range speed correlations near criticality on a system that develops such correlations for a different reason. We will test this alternative hypothesis in future work. Interestingly, our analysis also provides an approach for unveiling the relative importance of position-based and alignmentbased interactions in real systems. Indeed, the differences in their corresponding collective behavior, e.g. in their wave propagation properties, could be used to evaluate the relevance of each type of interaction in specific systems based only on their macroscopic dynamics.

Acknowledgments The work of $\mathrm{CH}$ was supported by the National Science Foundation under Grant No. PHY-0848755. The work of EF, AT and TW was supported by the Research Foundation Flanders (Flemish Community of Belgium) and European Science Foundation (ESF) funded H2Swarm project as well as by the KU Leuven funded BioCo3 IDO Project. The work of AT was supported by the Scientific and Technological Council of Turkey (TUBITAK) 2219 Program. We also acknowledge support by the Max Planck Institute for the Physics of Complex Systems in Dresden, Germany, through the Advanced Study Group "Statistical Physics of Collective Motion", where part of this work was conducted.

\section{References}

1. Attanasi, A., Cavagna, A., Del Castello, L., Giardina, I., Grigera, T.S., Jelic, A., Melillo, S., Parisi, L., Pohl, O., Shen, E., Viale, M.: Superfluid transport of information in turning flocks of starlings. arXiv:1303.7097 (2013)

2. Bak, P.: How Nature Works: The Science of Self-organized Criticality. Copernicus Series. Springer, Berlin (1999)

3. Ballerini, M., Cabibbo, N., Candelier, R., Cavagna, A., Cisbani, E., Giardina, I., Lecomte, V., Orlandi, A., Parisi, G., Procaccini, A., Viale, M., Zdravkovic, V.: Interaction ruling animal collective behavior depends on topological rather than metric distance: evidence from a field study. Proc Natl Acad Sci 105(4), 1232-1237 (2008)

4. Bialek, W., Cavagna, A., Giardina, I., Mora, T., Pohl, O., Silvestri, E., Viale, M., Walczak, A.M.: Social interactions dominate speed control in poising natural flocks near criticality. Proc Natl Acad Sci 111(20), 7212-7217 (2014)

5. Bialek, W., Cavagna, A., Giardina, I., Mora, T., Silvestri, E., Viale, M., Walczak, A.M.: Statistical mechanics for natural flocks of birds. Proc Natl Acad Sci 109(13), 4786-4791 (2012)

6. Binney, J.J., Dowrick, N.J., Fisher, A.J., Newman, M.E.J.: The Theory of Critical Phenomena: An Introduction to Renormalization Group. Oxford University Press, Oxford (1992)

7. Brambilla, M., Ferrante, E., Birattari, M., Dorigo, M.: Swarm robotics: a review from the swarm engineering perspective. Swarm Intell. 7, 1-41 (2013)

8. Breder, C.: Equations descriptive of fish schools and other animal aggregations. Ecology 35(3), 361-370 (1954)

9. Buhl, J., Sumpter, D., Couzin, I., Hale, J., Despland, E., Miller, E., Simpson, S.: From disorder to order in marching locusts. Science 312(5778), 1402-1406 (2006)

10. Buhl, J., Sword, G.A., Simpson, S.J.: Using field data to test locust migratory band collective movement models. Interface Focus 2(6), 757-763 (2012)

11. Cavagna, A., Cimarelli, A., Giardina, I., Parisi, G., Santagati, R., Stefanini, F., Tavarone, R.: From empirical data to inter-individual interactions: unveiling ther rules of collective animal behavior. Math. Models Methods Appl. Sci. 20(1), 1491-1510 (2010) 
12. Cavagna, A., Cimarelli, A., Giardina, I., Parisi, G., Santagati, R., Stefanini, F., Viale, M.: Scale-free correlations in starling flocks. Proc Natl Acad Sci 107(26), 11865-11870 (2010)

13. Cavagna, A., Giardina, I., Ginelli, F.: Boundary information inflow enhances correlation in flocking. Phys. Rev. Lett. 110, 168107 (2013)

14. Couzin, I.D., Krause, J., Franks, N.R., Levin, S.A.: Effective leadership and decision-making in animal groups on the move. Nature 433, 513-516 (2005)

15. Czirók, A., Stanley, H.E., Vicsek, T.: Spontaneously ordered motion of self-propelled particles. J. Phys. A 30(5), 1375 (1997)

16. Deutsch, A., Theraulaz, G., Vicsek, T.: Collective motion in biological systems. Interface Focus 2, 689 (2012)

17. Ferrante, E., Turgut, A.E., Dorigo, M., Huepe, C.: Collective motion dynamics of active solids and active crystals. New J. Phys. 15(9), 095011 (2013)

18. Ferrante, E., Turgut, A.E., Dorigo, M., Huepe, C.: Elasticity-based mechanism for the collective motion of self-propelled particles with springlike interactions: a model system for natural and artificial swarms. Phys. Rev. Lett. 111, 268302 (2013)

19. Ferrante, E., Turgut, A.E., Huepe, C., Stranieri, A., Pinciroli, C., Dorigo, M.: Self-organized flocking with a mobile robot swarm: a novel motion control method. Adapt. Behav. 20(6), 460-477 (2012)

20. Fetter, A., Walecka, J.: Theoretical Mechanics of Particles and Continua. Dover Books on Physics Series. Dover, Mineola (2003)

21. Flierl, G., Grünbaum, D., Levin, S., Olson, D.: From individuals to aggregations: the interplay between behavior and physics. J. Theor. Biol. 196, 397-454 (1999)

22. Gautrais, J., Ginelli, F., Fournier, R., Blanco, S., Soria, M., Chate, H., Theraulaz, G.: Deciphering interactions in moving animal groups. PLoS Comput. Biol. 8(9), e1002678 (2012)

23. Gautrais, J., Jost, C., Soria, M., Campo, A., Motsch, S., Fournier, R., Blanco, S., Theraulaz, G.: Analyzing fish movement as a persistent turning walker. J. Math. Biol. 58(3), 429-445 (2009)

24. Grossman, D., Aranson, I.S., Jacob, E.B.: Emergence of agent swarm migration and vortex formation through inelastic collisions. New J. Phys. 10, 023036 (2008)

25. Grunbaum, D., Okubo, A.: Modeling social animal aggregation. Frontiers in Theoretical Biology, Lecture notes in Biomathematics, vol. 100, pp. 296-325. Springer-Verlag, Berlin (1994)

26. Henkes, S., Fily, Y., Marchetti, M.C.: Active jamming: Self-propelled soft particles at high density. Phys. Rev. E 84(4), 040301(R) (2011)

27. Katz, Y., Tunstrm, K., Ioannou, C.C., Huepe, C., Couzin, I.D.: Inferring the structure and dynamics of interactions in schooling fish. Proc Natl Acad Sci 108(46), 18720-18725 (2011)

28. Kauffman, S.A.: The Origins of Order: Self-organization and Selection in Evolution. Oxford University Press, Oxford (1993)

29. Lewin, R.: Complexity: Life at the Edge of Chaos. Nature of Human Society Series. University of Chicago Press, Chicago (1999)

30. Lukeman, R., Li, Y.X., Edelstein-Keshet, L.: Inferring individual rules from collective behavior. Proc Natl Acad Sci 107(28), 12576-12580 (2010)

31. Menzel, M.A., Takao, O.: Soft deformable self-propelled particles. Europhys. Lett. 99(5), 58001 (2012)

32. Mora, T., Bialek, W.: Are biological systems poised at criticality? J. Stat. Phys. 144(2), 268-302 (2011)

33. Okubo, A.: Dynamical aspects of animal grouping: swarms, schools, flocks, and herds. Adv. Biophys. 22, 1-94 (1986)

34. Romanczuk, P., Couzin, I.D., Schimansky-Geier, L.: Collective motion due to individual escape and pursuit response. New J. Phys. 102(1), 010602 (2009)

35. Sumpter, D.J.T.: Collective Animal Behavior. Princeton University Press, Princeton (2010)

36. Szabó, B., Szöllösi, G.J., Gönci, B., Selmeczi, Z.J.D., Vicsek, T.: Phase transition in the collective migration of tissue cells: experiment and model. Phys. Rev. E 74(6), 061908 (2006)

37. Toner, J., Tu, Y.: Long-range order in a two-dimensional dynamical XY model: how birds fly together. Phys. Rev. Lett. 75, 4326-4329 (1995)

38. Toner, J., Tu, Y.: Flocks, herds, and schools: a quantitative theory of flocking. Phys. Rev. E 58, 4828-4858 (1998)

39. Turgut, A.E., Çelikkanat, H., Gökçe, F., Şahin, E.: Self-organized flocking in mobile robot swarms. Swarm Intell. 2(2), 97-120 (2008)

40. Vicsek, T., Czirok, A., Ben-Jacob, E., Cohen, I., Shochet, O.: Novel type of phase transition in a system of self-driven particles. Phys. Rev. Lett. 75(6), 1226 (1995)

41. Vicsek, T., Zafeiris, A.: Collective motion. Phys. Rep. 517(3-4), 71-140 (2012)

42. Warburton, K., Lazarus, J.: Tendency-distance models of social cohesion in animal groups. J. Theor. Biol. 150(4), 473-488 (1991) 